\title{
Merton's Jump Diffusion Model an Application to Stock Markets of East African Countries
}

\author{
Kimaro Novat ${ }^{1}$, Wilson Mahera Charles $^{2}$ and Verdiana Grace Masanja ${ }^{3}$ \\ ${ }^{1,3}$ The School of Computational and Communication Science and Engineering, the Nelson Mandela African \\ Institution of Science and Technology P.O. BOX 447, Arusha, Tanzania \\ ${ }^{2}$ The Department of Mathematics, University of Dar es Salaam P.O. Box 35062, Dar es Salaam, Tanzania
}

\begin{abstract}
The stock price is characterized with a number of features which can only be captured by a best model. To investigate this the Merton's jump diffusion model was applied to the selected stocks of three East African community countries' stock markets. The daily closing stock prices of the Nairobi Securities Exchange (NSE), the Dar es Salaam Stock Exchange (DSE) and Uganda Securities Exchange (USE) over a period of 5 years from 01/07/2013 to 01/07/2018 were analyzed with the objective of investigating how best the model captures the stock price features at these three East African stock markets. The Merton's jump diffusion model was considered as a stochastic differential equation and the Maximum Likelihood Estimation (MLE) method was used to estimate the optimal model parameters and implemented with MATLAB. The empirical results show that, the selected stocks from all the three markets exhibit a number of jumps as it was evidenced from non-zero values of jump intensities (lambda). Also, the log returns density of Merton reveals presence volatility and leptokurtosis features as evidenced by the presence of both negative and positive skewness and excessive kurtosis values.
\end{abstract}

Keywords: Volatility, Option Pricing, Leptokurtic, Lévy process, Jump diffusion models.

\section{INTRODUCTION}

Recently, a number of empirical studies point out that financial returns exhibit volatility with a stochastic pattern and fatter tails than the standard normal model, which has been shown to be unsuitable for capturing the asset price dynamics [1]. For some decades models such as the Black-Scholes model have been used in option pricing as a basis for capturing asset price dynamics [2]. The Black-Scholes approach assumes that the price of an asset, which is the underlying asset of the option follows a geometrical Brownian motion. But a geometrical Brownian motion cannot reflect most of aspects of a stock quotation [3]. Despite, the usefulness of Black- Scholes model, it fails to capture stock price features such as the leptokurtic and empirical abnormity called volatility smile [4].

However, several models have been proposed to accommodate such properties, particularly the leptokurtic and volatility smile[5]. Amongst others, these include the Model based on Lévy process [6] such as Merton Jump diffusion model [7], the Kou's jump-diffusion model and Variance Gamma Model [8]. Despite the availability and usefulness of these models, many have been applied to many of stock markets of the world in modelling stock prices rather than East African stock markets. Examples include, the study on the Japanese stock market using Kou Jump model [9], pricing options in jump diffusion using mellin transform at an American stock index of S\&P 500 [10], the study on Egypt, Nigeria and South Africa stock markets using ARJI-EGARCH model [11] and GARCH models to model stock returns volatility on Uganda Securities Exchange [12] , just to mention a few. 
International Journal of Advances in Scientific Research and Engineering (ijasre), Vol 5 (8), August-2019

Therefore, the main objective of this paper is to apply the Merton Jump Diffusion model to the stock markets of Nairobi Securities Exchange (NSE), Dar es Salaam Stock Exchange (DSE) and Uganda Securities Exchange (USE) to investigate if the model captures most of stock features basing on the daily closing stock price.

\section{METHODOLOGY}

\subsection{Assumptions and development of jump diffusion model}

The Merton's jump diffusion (MJD) model is built on Black-Scholes model by adding a compound Poisson jump process [13]. Additional of this jump process adds extra parameters $\lambda, \mu$ and $\delta$ to the original BS model which makes a total of five parameters. It is assumed that MJD model is an exponential Lévy model of the form, equation (1)

$$
S(t)=S(0) e^{L(t)}
$$

where, the stock price $(S(t) ; 0 \leq t \leq T)$ is modeled as exponential of Lévy process $(L(t) ; 0 \leq t \leq T)$. Merton's choice of the Lévy process is a Brownian motion with drift (continuous diffusion process) plus a compound Poisson process (discontinuous jump process) such that

$$
L(t)=\left(\alpha-\frac{\sigma^{2}}{2}-\lambda k\right) t+\sigma B_{t}+\sum_{m=1}^{N(t)} Y_{m}
$$

The log return is given by

$$
\ln \left(\frac{S(t)}{S(0)}\right)=L(t)=\left(\alpha-\frac{\sigma^{2}}{2}-\lambda k\right) t+\sigma B_{t}+\sum_{m=1}^{N(t)} Y_{m}
$$

where $\alpha$ denotes drift coefficient, $\sigma$ is denotes the volatility of diff usion, $(B(t) ; 0 \leq t \leq T)$ is the Standard

Brownian motion and the term $\sum_{m=1}^{N(t)} Y_{m}$ is a compound Poisson jump process with the normal distributed jumps $N(\mu, \delta)$ and the intensity denoted by $\lambda$.

\subsection{Model derivation}

The probability that an asset price jumps within a small time interval $d t$ can be written using a Poisson process $d N(t)$ as;

$$
\begin{gathered}
\operatorname{Pr}\{\text { an asset price jumps once in } d t\}=\operatorname{Pr}\{d N(t)=1\} \cong \lambda d t . \\
\operatorname{Pr}\{\text { an asset price jumps more than once in } d t\}=\operatorname{Pr}\{d N(t) \geq 2\} \cong 0 . \\
\operatorname{Pr}\{\text { an asset price does not jump in } d t\}=\operatorname{Pr}\{d N(t)=0\} \cong 1-\lambda d t .
\end{gathered}
$$

where the parameter $\lambda \in \mathbb{R}^{+}$is the intensity of jump process which is independent of time t.

If in the small time interval $d t$ the asset price jumps from $S(t)$ to $y_{t} S(t)$ for $y_{t}$, an absolute price jump size, so the relative price jump size (i.e. percentage change in the asset price caused by jump) is:

$$
\frac{d S(t)}{S(t)}=\frac{y_{t} S(t)-S(t)}{S(t)}=y_{t}-1
$$

It is assumes that the absolute price jump size $\left(y_{t}\right)$ is a nonnegative random variable drawn from a lognormal distribution, i.e. $\ln \left(y_{t}\right) \sim$ i.i.d. $N\left(\mu, \delta^{2}\right)$. This in turn implies that $E\left(y_{t}\right)=e^{\mu+\frac{1}{2} \delta^{2}}$ and $E\left[\left(y_{t}-E\left(y_{t}\right)^{2}\right]=\operatorname{var}\left[y_{t}\right]=\right.$ $e^{\mu+\frac{1}{2} \delta^{2}}\left(e^{\delta^{2}}-1\right)$. Incorporating these properties into the Merton's jump-diffusion dynamics of asset price, we obtain a SDE of the form;

$$
\frac{d S(t)}{S(t)}=(\alpha-\lambda k) d t+\sigma d B(t)+\left(y_{t}-1\right) d N(t) .
$$

Where $\alpha$ is the instantaneous expected return on the asset, $\sigma$ is the instantaneous volatility of the asset return conditional on that jump does not occur, $B(t)$ is the standard Brownian motion process and $N(t)$ is a Poisson process with intensity $\lambda$. 
International Journal of Advances in Scientific Research and Engineering (ijasre), Vol 5 (8), August-2019

It is assumed that $B(t), N(t)$ and $y_{t}$ are independent processes. Hence, the relative price jump size $y_{t}-1$ is log normally distributed with mean

$$
E\left[y_{t}-1\right]=e^{\mu+\frac{1}{2} \delta^{2}}-1=k
$$

and the variance

$$
E\left[\left(y_{t}-1-E\left(y_{t}-1\right)^{2}\right]=e^{2 \mu+\delta^{2}}\left(e^{\delta^{2}}-1\right) .\right.
$$

MJD model assumes that the log-return jump size $\ln \left(y_{t}\right) \equiv Y_{t}$ is a normal random variable such that

$$
\ln \left(\frac{y_{t} S(t)}{S(t)}\right)=Y_{t}=\ln \left(y_{t}\right) \sim i . i . d . N\left(\mu, \delta^{2}\right)
$$

\subsection{Solution to Merton's jump diffusion model}

Consider SDE in equation (5) and multiply by $S(t)$ on both sides then

$$
d S(t)=(\alpha-\lambda k) S(t) d t+\sigma S(t) d B(t)+\left(y_{t}-1\right) S(t) d N(t)
$$

From Itô formula for the jump-diffusion process given as:

$d f(X(t), t)=\frac{\partial f(X(t), t)}{\partial t} d t+b_{t} \frac{\partial f(X(t), t)}{\partial x} d t+\frac{\sigma_{t}{ }^{2}}{2} \frac{\partial^{2} f(X(t), t)}{\partial x^{2}} d t+\sigma_{t} \frac{\partial f(X(t), t)}{\partial x} d B(t)+[f(X(t)+\Delta X(t))-f(X(t))]$

Where, $b_{t}$ corresponds to the drift term and $\sigma_{t}$ corresponds to the volatility term of a jump-diffusion process and $f \in$ $C^{1,2}([0, T] \times \mathbb{R})$.

$$
\begin{aligned}
& \text { Let } f(S(t), t)=\ln (S(t)) \\
& \frac{\partial f}{\partial t}=0 \\
& \frac{\partial f}{\partial S}=\frac{1}{S(t)} \\
& \frac{\partial^{2} f}{\partial S^{2}}=-\frac{1}{S(t)^{2}}
\end{aligned}
$$

From equation (09)

$$
\begin{aligned}
b_{t} & =(\alpha-\lambda k) S(t) \\
\sigma_{t} & =\sigma S(t)
\end{aligned}
$$

Substituting the results from equations (12) to (16) into Itô formula in equation (10) then,

$$
\begin{gathered}
d \ln (S(t))=\frac{1}{S(t)}(\alpha-\lambda k) S(t) d t-\frac{(\sigma S(t))^{2}}{2} \times \frac{1}{S(t)^{2}} d t+\sigma S(t) \times \frac{1}{S(t)} d B(t)+\left[\ln \left(y_{t} S(t)\right)-\ln (S(t))\right] \\
d \ln (S(t))=(\alpha-\lambda k) d t-\frac{\sigma^{2}}{2} d t+\sigma d B(t)+\left[\ln \left(y_{t} S(t)\right)-\ln (S(t))\right] \\
d \ln (S(t))=\left(\alpha-\lambda k-\frac{\sigma^{2}}{2}\right) d t+\sigma d B(t)+\ln \left(y_{t}\right)
\end{gathered}
$$

Integrating equation (17) over the time interval $0 \leq s \leq t$, we get

$$
\int_{0}^{t} d \ln (S(s))=\int_{0}^{t}\left(\alpha-\lambda k-\frac{\sigma^{2}}{2}\right) d s+\int_{0}^{t} \sigma d B(s)+\sum_{m=1}^{N(t)} \ln y_{m}
$$




$$
\begin{gathered}
\left.\ln (S(t))\right|_{0} ^{t}=\left.\left(\alpha-\lambda k-\frac{\sigma^{2}}{2}\right) t\right|_{0} ^{t}+\left.\sigma B(t)\right|_{0} ^{t}+\sum_{m=1}^{N(t)} \ln y_{m} \\
\ln S(t)-\ln S(0)=\left(\alpha-\lambda k-\frac{\sigma^{2}}{2}\right) t+\sigma B(t)+\sum_{m=1}^{N_{t}} \ln y_{m} \\
\ln \left(\frac{S(t)}{S(0)}\right)=\left(\alpha-\lambda k-\frac{\sigma^{2}}{2}\right) t+\sigma B(t)+\sum_{m=1}^{N(t)} \ln y_{m}
\end{gathered}
$$

Recall, the definition of log price (return) jump size is $\ln y_{t} \equiv Y_{t}$

$$
\ln \left(\frac{S(t)}{S(0)}\right)=\left(\alpha-\lambda k-\frac{\sigma^{2}}{2}\right) t+\sigma B(t)+\sum_{m=1}^{N(t)} Y_{m}
$$

The probability density function (PDF) of the MJD-model log-return is

$$
\mathbb{P}\left(x_{t} \in A\right)=\sum_{i=0}^{\infty} \mathbb{P}(N(t)=i) \mathbb{P}\left(x_{t} \epsilon A \mid N(t)=i\right)
$$

Where, the term $\mathbb{P}(N(t)=i)=\frac{e^{-\lambda t}(\lambda t)^{i}}{i !}$ is the probability that the asset price jumps $i$ times during the time interval of length $t$ and $\mathbb{P}\left(x_{t} \epsilon A \mid N(t)=i\right)=\phi\left(x_{t} ;\left(\alpha-\lambda k-\frac{\sigma^{2}}{2}\right) t+i \mu, \sigma^{2} t+i \delta^{2}\right)$ is the Black-Scholes normal density of log-return assuming that the asset price jumps $i$ times in the interval of $\mathrm{t}$.

$$
\mathbb{P}\left(x_{t}\right)=\sum_{i=0}^{\infty} \frac{e^{-\lambda t}(\lambda t)^{i}}{i !} \phi\left(x_{t} ;\left(\alpha-\lambda k-\frac{\sigma^{2}}{2}\right) t+i \mu, \sigma^{2} t+i \delta^{2}\right) .
$$

\section{RESULTS AND DISCUSSION}

\subsection{Data}

Secondary data from DSE, NSE and USE were used. Data of the daily closing stock prices over a period of 5 years from 01/07/2013 to 01/07/2018 were used. Only 5 actively trading companies from each stock market were randomly chosen making a total of 15 companies from all the three stock markets. The trading days per year in all the three markets were assumed to be 252 days.

\subsection{Parameters estimation}

The algorithm for estimation of parameters the model in equation (18) is based on MLE method and was computed with MATLAB. The results of estimates of variables for each stock market are presented in tables 1,2 and 3.

Table 1: Estimation results of Merton's model for NSE

\begin{tabular}{|l|c|c|c|c|c|}
\hline Stock & $\hat{\alpha}$ & $\hat{\sigma}$ & $\hat{\lambda}$ & $\hat{\mu}$ & $\hat{\delta}$ \\
\hline KCB & 0.0442 & 0.1260 & 108.0856 & 0.0001 & 0.0193 \\
\hline KQ & -0.6293 & 0.1692 & 135.8863 & 0.0048 & 0.0355 \\
\hline LIMT & 1.3715 & 0.8509 & 4.2835 & -0.2260 & 0.000002 \\
\hline SCOM & 0.1584 & 0.0884 & 236.4803 & 0.0006 & 0.0138 \\
\hline C\&G & 0.1491 & 0.7388 & 50.3132 & -0.0156 & 0.1101 \\
\hline
\end{tabular}

Table 1, show that the daily jump intensity of The Safaricom Plc Ord 0.05 is very high which about 236 compared to other stocks of NSE. This indicates that stock is more sensitive to factors which affect the stock price. On other hand the daily jump intensity of the Limuru Tea Co.Plc Ord 20.00AIMS (LIMT) is about 4 which is relatively small compared to the rest. In general none of the stocks has zero value of jumping indicating that the price of these stocks vary randomly. The figure (1) for LIMT and figure (2) for SCOM illustrate this situation of jumps by considering the daily price and $\log$ return. 


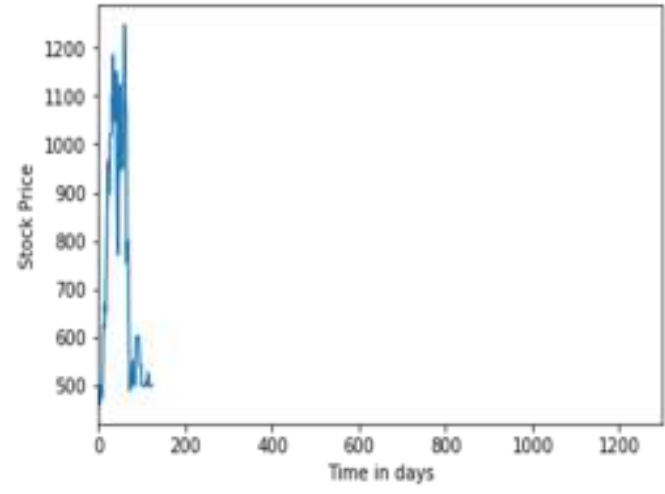

(a) The LIMT daily price level

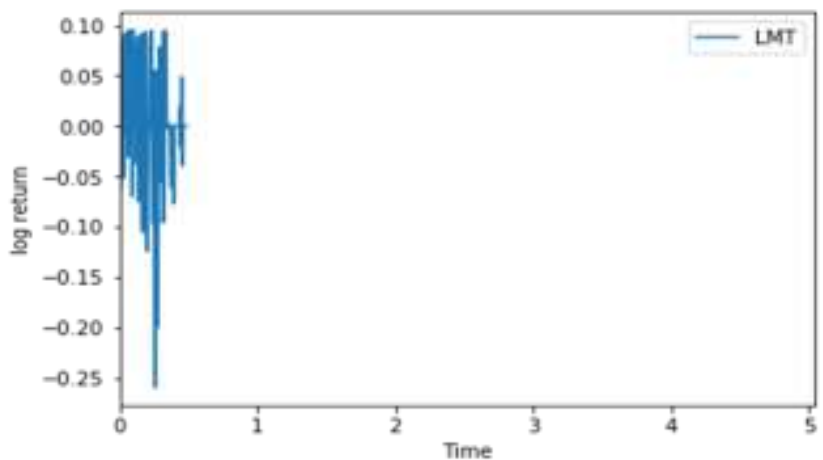

(b) The LIMT log return

Figure 1: The LIMT daily price level and log return (July, 2013 to July, 2018)

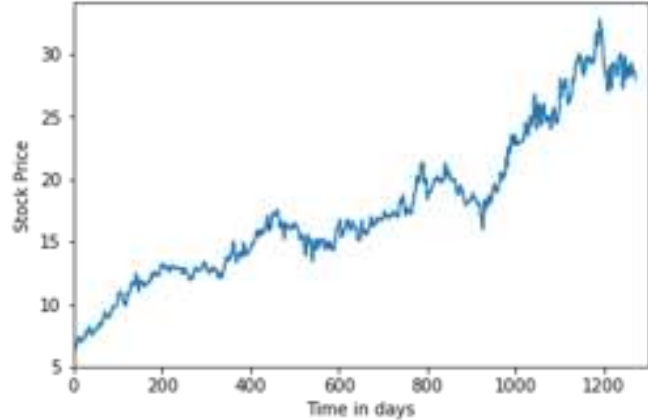

(a) The SCOM daily price level

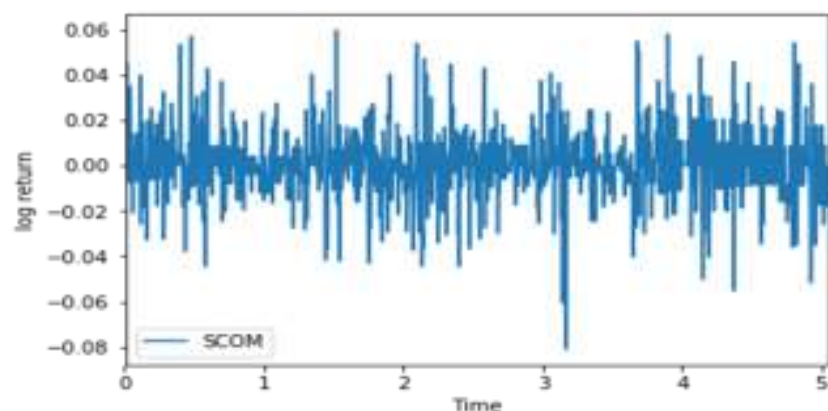

(b) The SCOM log return

Figure 2: The SCOM daily price level and log return (July, 2013 to July, 2018)

Table 2: Estimation results of Merton's Model for DSE

\begin{tabular}{|l|c|c|c|c|c|}
\hline STOCK & $\hat{\alpha}$ & $\hat{\sigma}$ & $\hat{\lambda}$ & $\hat{\mu}$ & $\hat{\delta}$ \\
\hline TBL & 0.1555 & 0.0333 & 14.9270 & 0.0187 & 0.0213 \\
\hline SWIS & 0.0641 & 0.0560 & 53.8672 & 0.0011 & 0.1284 \\
\hline NMB & 0.0853 & 0.2171 & 31.4789 & 0.0039 & 0.0125 \\
\hline TCC & 0.1941 & 0.1983 & 9.7657 & 0.0047 & 0.4353 \\
\hline VODA & -0.1154 & 0.2098 & 1.1156 & -0.0251 & 0.0991 \\
\hline
\end{tabular}

The results in table 2, show that the jump intensities of Tanzania Breweries (TBL), Vodacom Tanzania (VODA) and Tanzania Cigarette Company (TCC) are relatively small compared to Swissport Tanzania (SWIS) and NMB Bank Plc (NMB) .However, the expected returns for all stocks are positive except VODA which has a negative rate of expected rate of return of about $12 \%$. 


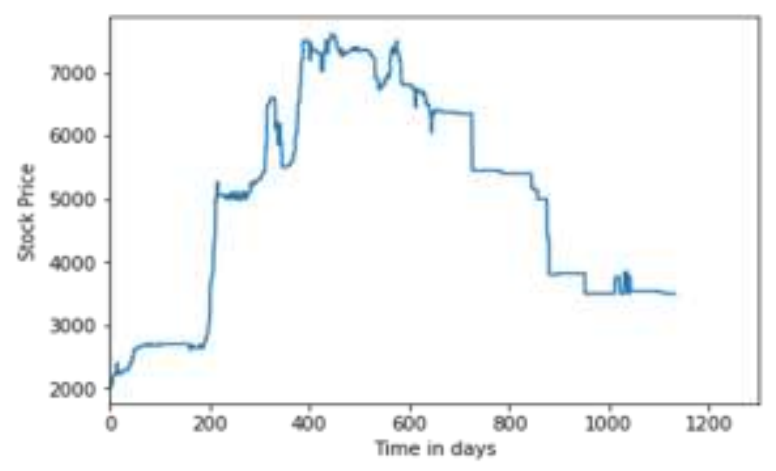

(a) The SWIS daily stock price level

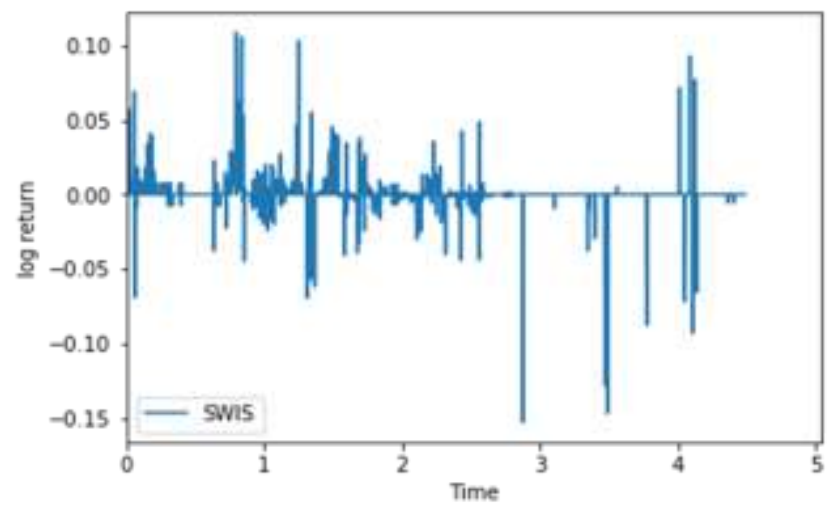

(b) The SWIS log return

Figure 3: The SWIS daily price level and log return (July, 2013 to July, 2018)

The price of SWIS as shown in figure (3a) and (3b) its movement is random and that the log return jump is high compared to the rest of five stocks of DSE.

Table 3: Estimation results of Merton's Model for USE

\begin{tabular}{|l|c|c|c|c|c|}
\hline STOCK & $\hat{\alpha}$ & $\hat{\sigma}$ & $\hat{\lambda}$ & $\hat{\mu}$ & $\hat{\delta}$ \\
\hline BATU & 0.0094 & 0.0181 & 16.0868 & 0.1314 & 0.1396 \\
\hline BOBU & -0.0275 & 0.0506 & 11.0200 & 0.0054 & 0.0516 \\
\hline NIC & 0.0219 & 0.0215 & 71.1078 & -0.0054 & 0.1900 \\
\hline UMEM & -0.0108 & 0.0725 & 55.1214 & 0.0004 & 0.0774 \\
\hline SBU & 0.0106 & 0.0390 & 84.6321 & 0.0002 & 0.2570 \\
\hline
\end{tabular}

Table 3 shows that, the stock price of Stanbic Bank Uganda (SBU) has high jump intensity among all five stocks of USE. The Bank of Baroda Uganda (BOBU) and UMEME LIMITED (UMEM) both have a decreasing return compared to the remaining three stocks. On other hand, the BOBU is having very small jump among other five stocks of USE.

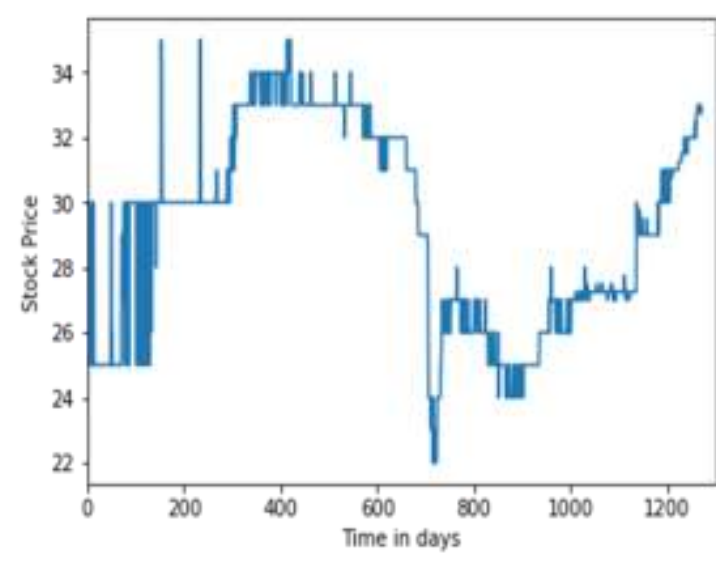

(a) The SBU daily stock price level

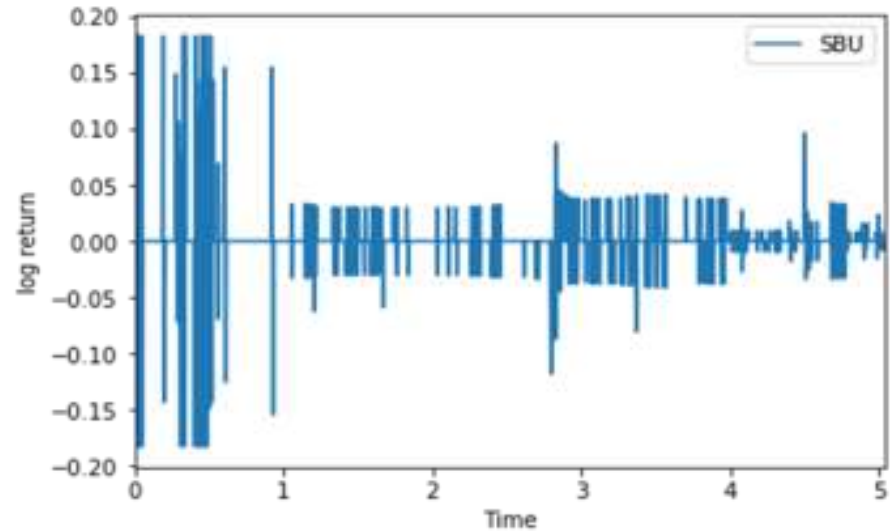

(b) The SBU log return

Figure 4: The SBU daily price level and log return (July, 2013 to July, 2018) 
Figures (4a) and (4b) show the SBU daily price level and log return respectively, the ups and downs (jumps) are very close in both figures. The daily movement of stock price in figure (4a) is random, likewise for the log return in figure (4b).

In tables 1, 2 and 3 it can be concluded that the jump intensity for all stocks is not zero justifying that the stock price exhibit the jumps. Also the stock in all three markets seems to have nearly similar properties especially the instantaneous return this is because in all three markets the returns for some stocks are positive indicating the increasing return, while others have negative values indicating a decreasing return.

\subsection{The Moments of Merton's log return density}

The table 4 summarizes the moments of Merton's log return density.

Table 4: The mean, variance, skewness and kurtosis of Merton's log return density for all selected stocks

\begin{tabular}{|c|c|c|c|c|}
\hline Stock & Mean & Variance & Skewness & Kurtosis \\
\hline KCB & -0.0003 & 0.0002 & 0.0144 & 6.5977 \\
\hline KQ & -0.0006 & 0.0008 & 0.4306 & 7.1041 \\
\hline LIMT & -0.0018 & 0.0037 & -0.8574 & 6.1680 \\
\hline SCOM & 0.0008 & 0.0002 & 0.1057 & 5.3227 \\
\hline C\&G & -0.0055 & 0.0046 & -0.3614 & 7.2623 \\
\hline TBL & 0.0016 & 0.00005 & 5.0553 & 40.0757 \\
\hline SWIS & 0.00030 & 0.0035 & 0.0553 & 16.9359 \\
\hline NMB & -0.00004 & 0.0002 & 0.0783 & 3.2522 \\
\hline TCC & 0.0002 & 0.0075 & 0.1594 & 77.2260 \\
\hline VODA & -0.0014 & 0.0002 & -1.0182 & 32.6454 \\
\hline BATU & 0.0084 & 0.0023 & 5.5848 & 43.0367 \\
\hline NIC & -0.0015 & 0.0102 & -0.1603 & 13.6279 \\
\hline UMEM & -0.0002 & 0.0013 & 0.0324 & 16.2888 \\
\hline BOBU & -0.0007 & 0.0001 & 1.3093 & 61.1292 \\
\hline SBU & -0.00005 & 0.0222 & 0.0040 & 11.9279 \\
\hline
\end{tabular}

Table 4 shows that, with exception of Car \& General (K) Ltd Ord 5.00 (C\&G), Vodacom Tanzania (VODA) and National Insurance Corporation (NIC), the Limuru Tea Co. Plc Ord 20.00AIMS (LIMT) which are negative skewed the rest of stocks are positive skewed. The presence of skewness indicates that the stock prices have non-symmetric return and exhibit empirical abnormity called volatility smile. The volatility measures the degree of variation of a trading price series over time as it is measured by standard deviation of log returns. Also in table (4), NMB Bank Plc (NMB) has very small kurtosis value of about 3.2 among all selected stocks at all three markets, likewise TCC has very high kurtosis value of about 77 compare to other stocks. Kurtosis signifies the presence of the leptokurtic features (fatter tails and high pick than normal curves). 


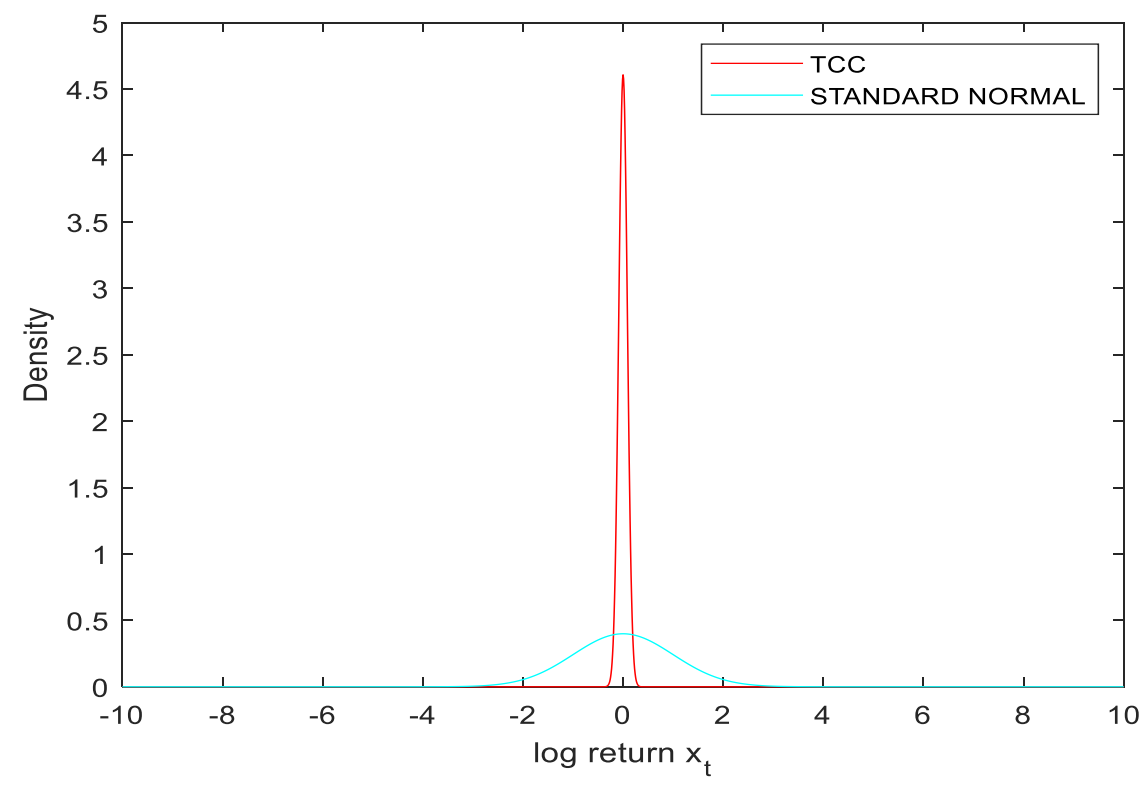

Figures 5: The comparison of kurtosis for empirical TCC and standard normal

Figure (5), shows TCC in red with high pick than the standard normal distribution in cyan. The kurtosis value for all of stocks is greater than three compared to the normal distribution which is not supposed to exceed three. This shows that, the underlying distribution of the returns are leptokurtic or heavy tailed as it can be seen on the figure (5)

\section{CONCLUSION}

Generally, basing on the established empirical results, the following can be concluded. Firstly, this study has established that the stocks from all three markets exhibit a number of jumps as it can be evidenced from the figures and numerical values from the tables. Secondly, the log returns density reveal volatility and leptokurtosis as evidenced from the presence of skewness and kurtosis values. Lastly but not least it is now suggested that the Merton's jump diffusion model fits well stock prices data of East African stock markets as it has captured most of key features of stock prices.

\section{ACKNOWLEDGEMENT}

The authors acknowledge the Nelson Mandela African Institution of Science and Technology for work environment and facilities which facilitated the study and the writing of this article. Additionally, the authors acknowledge the African Development Bank (AfDB) for funding.

\section{REFERENCES}

1. Costabile.M, Leccadito.A, Massabó.I and Russo.E. "Option pricing under regime-switching jump-diffusion models," J. Comput. Appl. Math., vol. 256, pp. 152-167, Jan. 2014

2. Yi, L. The Pricing of Options with Jump Diffusion and Stochastic Volatility. Iceland, 2010

3. Burger.P and Kliaras.M. Jump Diffusion Models for Option pricing vs.Black Scholes Model. (81), 1-73, 2013

4. Kou, S. G. Jump-Diffusion Models for Asset Pricing in Financial Engineering. 15(07), 73-116, 2008. https://doi.org/10.1016/S0927-0507(07)15002-7

5. Lin, X.C, Miao, D.W, Chao, Wand, D. Miao.W ."Analysis of a Jump-Diffusion Option Pricing Model with Serially Correlated Jump Sizes Analysis of a Jump-Diffusion Option Pricing Model with Serially Correlated Jump Sizes," vol. 0926, no. April, 2017.

6. Cont.R and Tankov.P. Financial Modelling With Jump Processes. Chapman Hall/CRC Press, 2004.

7. Merton.R.C. "Option Pricing When underlying stock returns are discontinuous," J. financ. econ., vol. 3, no. 1-2, pp. 125-144, 1976.

8. Matsuda.K. "Introduction to Merton Jump Diffusion Model," no. 1999, pp. 1-26, 2004. 
9. Maekawa.K, Lee.S, Morimoto.T and Kawai.K."Jump diffusion model with application to the Japanese stock market," vol. 78, pp. 223-236, 2008.

10. Frontczak.R. "Pricing Options in Jump Diffusion Models Using Mellin Transforms," J. Math. Financ., vol. 3, no. 3, pp. 366-373, 2013.

11. Kuttu.S. "Time-varying conditional discrete jumps in emerging African equity markets," Glob. Financ. J., vol. 32, pp. 35-54, 2017.

12. Namugaya.J, Weke.P, and Charles.W.M, "Modelling Stock Returns Volatility on Uganda Securities Exchange," vol. 8, no. 104, pp. 5173-5184, 2014.

13. Matsuda.K. "Introduction to Option Pricing with Fourier Transform: Option Pricing with Exponential Lévy Models," no. December, 2004. 